\title{
The University of South Florida homograph norms
}

\author{
DOUGLAS L. NELSON, CATHY L. McEVOY, JOHN R. WALLING, and \\ JOSEPH W. WHEELER, JR. \\ University of South Florida, Tampa, Florida 33620
}

Norms were collected to determine the relative dominance of different meanings of homographic words. Forty-six subjects wrote down the first word that came to mind for each of 320 homographs. Each homograph, the number of times each meaning was given, and the specific associates are made available. In addition, correlations with other norms are presented.

Homographs are words with identical spellings and two or more distinct meanings. They make ideal stimuli for research on a variety of problems (cf. Wollen, Cox, Coahran, Shea, \& Kirby, 1980). However, in the absence of context, some semantic interpretations are more likely to occur than others. Therefore, it is important to have some estimate of meaning dominance before these stimuli can be used in research. Several sets of normative data are available, but they include only 40-117 homographs and often present only primary and secondary associates (Kausler \& Kollasch, 1970) or no associates at all (Geis \& Winograd, 1974; Wollen et al., 1980). Because associates are frequently used as retrieval cues or as priming items for homographic words (e.g., Nelson, Walling, \& McEvoy, 1979; Schvaneveldt \& Meyer, 1973), their availability is essential. Prompted by the obvious need for this information, the present effort to norm a large sample of homographs was initiated in 1976.

\section{METHOD}

\section{Materials}

The 320 words appearing in the norming task were all selected from Roget's International Thesaurus (1962). Each had at least two distinct meanings that were judged as likely to be understood by everyone. As shown in Table 1,53 of the homographs in these norms overlapped with the 100 used by Cramer (1970), 39 overlapped with the 40 used by Kausler and Kollasch (1970), 74 with the 109 used by Perfetti, Lindsey, and Garson (1971), 30 with the 50 of Geis and Winograd (1974), and 67 with the 117 of Wollen et al. (1980).

\section{Subjects}

The subjects were 46 introductory psychology students who participated on a volunteer basis for class credit.

Procedure

All words were typed in uppercase letters and in random

This research was supported by Grant MH 16360 to the first author from the National Institute of Mental Health. Requests for reprints should be sent to Douglas L. Nelson, Department of Psychology, University of South Florida, Tampa, Florida 33620.
Table 1

Available Homograph Norms, Number of Items Evaluated, Number of Items Overlapping With the Present Norms, and Correlations Between the Present and Other Norms

\begin{tabular}{lccc}
\hline & \multicolumn{3}{c}{ Number } \\
Norms & of Items & Overlapping & r \\
\hline Cramer (1970) & 100 & 53 & .79 \\
Kausler and Kollasch (1970) & 40 & 39 & .88 \\
Perfetti et al. (1971) & 109 & 74 & .86 \\
Geis and Winograd (1974) & 50 & 30 & .91 \\
Wollen et al. (1980) & 117 & 67 & .79 \\
Nelson et al. (1980) & 320 & & \\
\hline
\end{tabular}

order on eight pages, with 40 words/page. Each set of 40 words was arranged into two vertical columns. Booklets were then constructed by unsystematically randomizing the order of the eight pages. The instructions were presented both orally and printed on the cover sheet of the booklet, as follows.

"This booklet contains over 300 words. Next to each word is a blank. When I say 'begin' your task will be to write next to each word the first word that it makes you think of. It doesn't make any difference what word you write as long as the word on the paper makes you think of it. There are no right or wrong answers. For example, if the word was 'BAWL' you might write 'CRY'. If the word was 'DOG' you might write 'CAT' or 'HOUND'. The proper way of indicating this word is:

$\begin{array}{ll}\text { BAWL } & \text { CRY } \\ \text { DOG } & \text { HOUND }\end{array}$

Be sure to write or print your word clearly and don't worry if you are not sure how to spell it. Spell the word as best as you can. Work as fast as possible, writing only a single word in each blank."

All subjects completed the task within the alloted 50 -min period. Note that the instructions never mentioned the homographic nature of the stimuli.

\section{RESULTS AND DISCUSSION}

Separate categories were made for each homograph, including all the responses given to each word and their frequencies. Four judges (the authors) worked individually to categorize each response according to the meaning most likely to produce it. Then, before final categorization, each response was discussed until a 
consensus was achieved. The semantic categories were chosen to reflect the origin of each meaning of the word according to The American Heritage Dictionary of the American Language (1973). When two closely related meanings came from the same source, they were treated as a single category. If separating two related meanings resulted in many responses that could be assigned to either category, the meanings were combined (e.g., the "dollar" and "statement" meanings of BILL were combined under "money"). Words referring to both an object and an action involving that object (e.g., DRILL) were included in a single category. Furthermore, a response that could not be classified with any of the meanings or that was obviously the result of a misinterpretation was placed in the questionable (?) category. Finally, since all responses are reported, anyone wishing to disagree with our judgments can readily recategorize the items.

Pearson product-moment correlations were calculated between our norms and others using homographs common to both sets. Before calculating these correlations, meanings were combined when necessary to coordinate categories in different norms, and, therefore, these correlations need to be regarded as approximate. All values were converted to proportions and averaged over sex of the subject. Both dominant and nondominant meanings were included. Thus 106 pairs of proportions were used in calculating the $r$ with the Cramer (1970) norms. Similarly, 60, 83, 186, and 148 pairings were used in calculating the respective rs for the Geis and Winograd (1974), Kausler and Kollasch (1970), Perfetti et al. (1971), and Wollen et al. (1980) norms.

The results of this correlational analysis are shown in Table 1. All of the values appear to be fairly high and agree quite well with those reported by Wollen et al. (1980). This agreement is obtained despite differences in subjects, sample size, evaluating judges, geographic region, and, finally, despite differences in procedure. Our norms and those of Cramer (1970) both employed the "first response" technique and correlated .79. Wollen et al. used short phrases and careful control of the timing arrangements, and, despite these differences, our values correlated .79 with theirs. Apparently, greater control does not substantially alter the proportions of responses allocated to the various meanings.

Table 2 presents each homograph listed in alphabetical order. The meanings, or senses, of each item are listed directly under it, and, in parentheses, the number of subjects providing responses with that meaning is given. As indicated, the ? category was used only when no other possible meaning could be determined. The N/R category represents "no responses," or omissions. Note that the individual responses (and their frequencies) are shown to the right of each meaning.

Table 2

Frequency of Occurrence of Each Meaning and Each Response

$\begin{array}{ll}\text { ARM } & \\ \text { Limb (43) } & \begin{array}{l}\text { leg (23), hand (7), hammer (3), pit (2) } \\ \text { appendage, crook, in, write, build } \\ \text { band, shoulder, around }\end{array} \\ \text { Weapon (1) } & \text { war } \\ ?(1) & \text { farm } \\ \text { N/R (1) } & \end{array}$

BALL

Round object (42)

Dance (1)

Sex (1)

?(1)

N/R (1)

BANK

Money (45)

? (1)

BAR

Drink (35) baseball, pool, foot, hit

dance

screw

call Union Trust

tank
Oblong object $(6)$

Exclude (2)

Law (1)

?(2)

BARK

Dog (37)

Tree (7)

?(1)

N/R (1)

game (6), base (4), round (4), bounce
(3), throw (3), play (3), bat (3), point, basketball, red, rubber, square, bearing, stick, park, fun, catch, chain, pen,

money (20), robbery (8), account (3), teller (2), statement, savings, roll, hours, trust, deposit, Tampa, card, bureaucracy, vault, save, Landmark

BAT

drink (12), beer (4), tavern (2), stool (2), booze (2), lounge (2), liquor (2), redneck, Nebraska Ave., order, juice, tender, hop, maid, drunk, rum

BASE

Baseball (28)

Foundation (11)

Headquarters (2)

Chemical (2)

? (3)

BASS

Fish (27)

Music (17)

Stick (24)

Animal (20) crow, bell, handle, gold, press, steel keep, it

law

three, far

$\operatorname{dog}(32)$, loud (3), bite, howl

tree (7)

fish ball (19), line (4), home (2), safe, first, hit

bottom (5), foundation, ground, seat, lamp, low, number

station, army

strong, acid

fiddle, face, case

fish (26), trout

drum (3), fiddle (2), guitar (2), treble (2), deep (2), instrument, vial, tuba, tenor, cello, music

grandmother, mass

ball (2), boy (3), baseball

fly (4), rat (2), cat (2), cave (2), radar, man, dark, cry, wing, animal, bird, dangerous, rabies, vampire 
Table 2 (Continued)

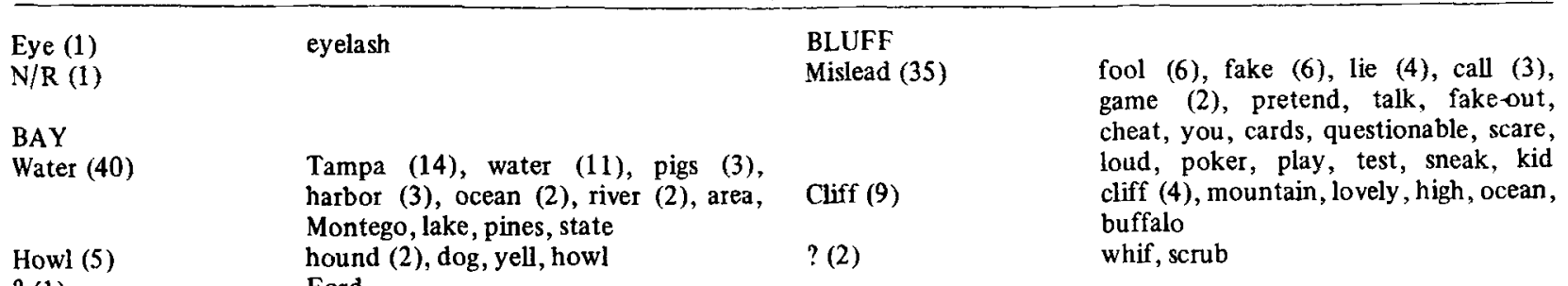

? (1)

Ford

BEAR

Animal (42)

Pressure (3)

?(1)

BEAM

Construction (26)

Light (17)

Whiskey (2)

N/R (1)

BEEF

Meat (46)

BILL

Money (37)

Name (7)

Proposed law (1)

? (1)

BLOCK

Obstacle (21)

Flat sided substance (15)

City (9)

?(1)

\section{BLOW}

Air (42)

Hit (3)

N/R (1)

BLUE

Color (45)

balls

fill clue animal (8), woods (3), cub (3), brown (3), grizzly (3), big (3), skin (2), black (2), polar (2), hug (2), furry, growl, lion, Pan, forest, rifle, coats, rug, hog, hunt, yogi

weight, down, wear

\section{BOARD}

Plank (24)

Display (13)

Directors (7)

? (2)

BOLT

wood (6), ceiling (4), house (3), wooden (2), building (2), support, board, weight, overhead, balance, ship, rod, steel, exposed

light (12), laser, sun, joy, radiate, gleam

$\operatorname{Jim}(2)$

Fastener (29)

Sudden movement (7)

Lightning (5)

Cloth (2)

? (3)

BOOT

Footwear (44)

cow (10), steak (9), stew (8), cattle (3), eat (3), meat (2), steer (2), brown, eater, expensive, ground, pork, animal, veal, angus, meat

pay (7), money (5), payment (2), paid (2), me (2), check (2), collector (2), dollar (2), fold, creditors, cost, tax, sale, affair, statement, owe, of fare, goods, lading, bond, green

John, Uncle, guy, name, person, Jane, father

government

stop (5), tackle (3), road (2), buster (2), shot, with, mental, strike, thrust, football, pass, defense, obstruct wood (7), ice (3), head (2), cement, concrete, build

city (3), street (2), cell (2), house (2) apple

horn (16), wind (11), hard (6), whistle (2), down (2), up, tired, exhale, balloon, air

hit (2), mistake

sky (9), red (8), green (8), color (5), water (3), white (2), yellow (2), eyes (2), grey, sea, boy, black, ink, night
New recruit (1)

?(1)

\section{BOUND}

Tied $(25)$

Jump (13)

Headed for (6)

BOW

Arrow (26)

Bend (10)

Tie (7)

Ship (2)

N/R (1)

BOWL

Container (23)

Ball (20)

N/R (3)

BOX

Container (33) wood (8), plank (4), wooden (2), ship (2), nails, surf, long, slat, walk, dive, build, stiff

chalk (4), black (3), bill (2), game (2), blackboard, bulletin

directors (2), room (2), rules, chairman, regents

closet, tired

nut (11), door (6), lock (4), screw (3), close (2), stove, dead, steel

jump (2), run (2), leap, upright, colt lightning (5)

cloth, material

neck, holt, game

shoe (15), kick (6), leg (5), leather (4), foot (3), lick (3), strap (3), winter, black, cowboy, ass, skate

camp

hoot

tied (8), tie (4), gagged (3), rope, tieup, chained, unbound, overwatch, held, closed, bind, tape, book

jump (5), leap (4), bounce, up, over, hop

home (2), determined, homeward, travel, goal

sound, found

arrow (25), archery

curtsey (2), respect, stoop, bless, out, karate, bend, boy, king

tie (6), gift

boat, stern

cereal (5), dish (2), soup (2), salad (2), spoon, jelly, cup, breakfast, toilet, broken, food, cherries, rice, dog, oatmeal, pan

ball (7), pins (4), game (3), dollars, sport, goal, alley, rose, strike

square (5), lunch (4), cardboard (3), car (3), top (2), container (2), crate, black, Skinner, shoe, open, wood, in, keep, mail, stack, cigars, seat, spring, vagina 
Office (4)
Fight (6)
$?(2)$
N/R (1)
BREAK
Ruin (33)

Escape (11)

Rest (2)

BRIDGE

Span (28)

Cards (16)

N/R (2)

BRIDGE

Span (31)

Cards (14)

?(1)

BUG

Insect (38)

Bother (6)

Car (2)

CABLE

Heavy wire (40)

? (4)

N/R (2)

CALL

Summon (43)

Stop (2)

N/R (1)

CAN

Container (29)

Ability (8)

Dance (5)

Fire (2)

$N / R(2)$

\section{CANE}

Plant (21)

Stick (21) office (4)

hit (4), play, boxer

camille, fox

nin (3), broken (3), fix (2), tear (2), repair (2), open, down, splatter, up, shatter, mend, crack, crash, bottle, bone, egg, smash, fit, bend, neck, glass, destroy, snap, hurt, heart, bust

out (2), even (2), away (2), jail (2), fast (2), thru

intermission, coffee

water (7), over (4), span (2), river (2), steel (2), work, cross, troubled water, gap, Kwai, demolition, build, tunnel, London, wooden, teeth

game (7), cards (6), club (2), play

water (11), river (3), gap (2), London (2), span (3), Kwai, cross, over, across, high, Washington, builder, steel, wooden, nose

cards (8), game (4), partner, party ridge

insect (11), roach (7), spray (4), beetle (3), mosquito, kill, ladybug, screen, spider, net, yech!, squash, bad, love, bite, small, fly

bother (3), off (2), out

VW, car

car (12), telephone (5), line (4), wire (3), TV (3), call, train, cord, rope, splice, bridge, telegraph, telegram, release, ocean, pull, steel, jack

Clark (2), table, marble

phone (16), girl (6), yell (5), wild (3), again (2), card, dog, shout, name, number, hail, parent, on, me, friend, hey ball (2)

tin (4), opener (4), tuna (2), food (2), soup (2), goods (2), bottle (2), container (2), peaches, jar, beer, liá, empty, open, beef, beans, drink will (3), can't, not, do, able, yes can (5)

fire (2)

sugar (21)

walk (5), blind (4), stick (3), candy (2), pole (2), old, man, cripple, support, crutches
? (4)

CAP

Hat (26)

Top (11)

? (4)

N/R (5)

CARDS

Playing (39)

Greeting (4)

?(1)

$\mathrm{N} / \mathrm{R}(2)$

\section{CARP}

Fish (38)

Nag (4)

$?(3)$

N/R (1)

CASE

Container (24)

File (16)

Protective covering (1)

? (2)

N/R (3)

CAST

Dressing (15)

Throw (14)

Theater (10)

Iron (5)

? (2)

CELL

Jail (27)

mutiny (2), cane, Abel

hat (17), head (4), gown (2), night, glove, baseball

ground (3), top (2), enrollment, pen, gas, on, stopup, screw

crew, sap, grass, gap

poker (10), deck (7), game(s) (7), play (7), ace (2), gambling, rummy, shuffle, spade, king, playing

greeting, paper, post, read

silence

fish (37), trout

harp (2), anger, nag

port, carcass, tarp

book (6), beer (5), container (2), brief (2), box (2), valise, guitar, gun, crate, glass, watch, jacket

history (3), load (2), jury, suit, study, work, joint, court, number, lawyer, file, law, closed

harden

one, base

leg (6), broken (5), arm (2), broken leg, break

fish (5), throw (4), doubt, sling, aside, off, shadow

show (2), actors, thousands, big, stage, group, assign, broadway, play

iron (3), mold, built

fast, totem

fail (10), prison (5), block (3), walls (2), room, closed, lock, man, dark, empty, confined

Bìological (16)

body (3), blood (2), biology (2), small (2), amoeba (2), nucleus, one-celled, unit, organism, factor

Battery (1)

? (2)

dry

jello, bell

CHANGE

Money (25)

Alter (21)

money (16), coins (3), quarter (2), small money, fare, dollar, pennies different (2), ways, -ling, it, clothes, tire, alter, begin, mind, revamp, relocate, coming, direction, transition, battery, grow, move, switch, strange, metamorphosis

CHARGE

Payment (35)

account (7), card (7), credit (4), it (3), cost (3), cash (2), admission (2), credit card, interest, price, fare, fee, master, money 
Table 2 (Continued)

Attack (5)

Accusation (3)

Electricity (2)

? (1)

\section{CHARM}

Attract (25)

Trinket (15)

Omen (3)

? (2)

N/R (1)

\section{CHECK}

Bank (30)

Verify (12)

Chess (4)

\section{CHEST}

Body (23)

Treasure (11)

Bureau (6)

? (6)

CHEW

Teeth (44)

Scold (2)

CHINA

Dishes (22)

Country (16)

Doll (7)

N/R (1)

\section{CHOP}

Meat (24)

Cut (13)

Wood (7)

N/R (2)

CHUCK

Meat (24)

Throw (11)

Wagon (7)

Name (4)

\section{CLUB}

Organization (19) force, fun, move, rush, Teddy Roosevelt arrest, blame, rape

battery, wire

nurse

smile (4), poise (2), grace (2), school (2), wit (2), charisma (2), warm, perfume, appealing, like, Carter, nice, class, look, elegance, women, pleasant bracelet (10), metal, doll, ring, jewel, necklace

harm, omen, luck

Shehag, farm

money (13), book (8), cash (3), bounce (3), write (2), bank

list (5), out (3), point (2), mark, double mate (4)

heart (3), hairy (2), big (2), breast (2), body (2), X-ray (2), bosom, torso, power, shirt, breathe, cold, pain, stomach, pride, hair

treasure (6), box (2), store, container, lock

drawers (4), drawer, mirror

game (3), board, play, nest

food (10), gum (8), eat (8), mouth (4), teeth (3), masticate (3), meat (2), bite (2), swallow, cud, tobacco, fat

out, over

dishes (8), cabinet (3), ware (3), plates (3), Wedgewood, shop, bowl, bone, service

country (5), Nixon (3), town (3), Red (2), Israel, Japan, house doll (7)

pork (10), meat (4), steak (4), lamb (3), liver (2), bite

cut (4), suey (4), down (2), sticks, up, grate

wood (3), hit (3), hatchet

steak (12), roast (7), beef (3), stew, ground

throw (9), over, throw out

wagon (7)

name, who, despise, Snoopy

country (2), people (2), clique, fraternity, 4-H, mix, group, join, boys, peer, money, organization, belong, dinners, member, bridge, gentleman
Glide (4)

? (1)

$\mathrm{N} / \mathrm{R}(2)$

COAT

Weapon (10)

Hit (8)

Room (5)

Foods (3)

Cards (1)

COAST

Shore (39)

Jacket (40)

Covering (4)

Animal (1)

?(1)

COMB

Hair (44)

Search (1)

N/R (1)

CORD

Rope (41)

Wood (3)

? (1)

N/R (1)

CORN

Grain (46)

COUNT

Number (39)

Nobleman (5)

Depend (1)

?(1)

COURT

Legal (33)

Uncovered space (8)

Sovereign (2)

Romance (2)

? (1)

CRAB

Shellfish (45) stick (5), golf, billy, cane, bat, weapon hit (8)

house (4), lounge

sandwich (2), soda

heart

guard (7), water (5), beach (4), west (4), east (3), Florida (2), shore (2), line (2), shoreline, edge, land, gold, country, California, reef, sunshine, Cocoa Beach, clear

slide, glide, ride, relax

toast

hat (6), warm (6), fur (5), hanger (4), tie (4), gold (3), jacket (3), pocket (2), wear, mink, garment, gloves, clothes, winter, mail

cover (2), colors, edge

cat

boat

hair (30), brush (13), fix

search

rope (6), string (6), tie (6), electric (4), rip (4), lamp (3), light (2), wires (2), extension (2), outlet, pull, line, knot, hanging, umbilical

wood (2), board

guitar

cob (10), kernel (6), husk (4), ear (4), vegetable (3), yellow (2), field (2), farmer, bread, food, crop, dog, shuck, pop, whiskey, beef, stack, meal, starch, eat, grits, peas

numbers (24), down (5), money (4), ten (2), counter, up, head, two

Dracula (4), Duke

depend

tell

judge (5), room (5), law (6), date (2), trial (2), jury (2), house, jail, county, justice, action, bail, fine, marshall, crime, busted, case

yard (5), tennis (3)

jester, kings

lady, ship

coat

meat (11), fish (3), shell (3), crustacean (2), water (2), claw (2), beach (2), sea (2), lobster (2), food, cracked, lice, 


\begin{tabular}{|c|c|}
\hline Crabtree (1) & $\begin{array}{l}\text { fiddler, shore, eat, animal, salad, ocean- } \\
\text { ography, cake, legs, good, back, king, } \\
\text { deviled, roll } \\
\text { tree }\end{array}$ \\
\hline \multicolumn{2}{|l|}{ CROOK } \\
\hline Thief (42) & $\begin{array}{l}\text { thief (12), steal (6), robber (5), crime } \\
\text { bank (2), rob ( } 2) \text {, criminal ( } 2) \text {, crack, } \\
\text { shoot, cop, dishonest, bad guy, pris- } \\
\text { oner, bad, bastard, scoundrel, Nixon, } \\
\text { gangster }\end{array}$ \\
\hline $\begin{array}{l}\text { Curve (2) } \\
?(2)\end{array}$ & $\begin{array}{l}\text { arm, finger } \\
\text { no, pickles }\end{array}$ \\
\hline \multicolumn{2}{|l|}{ CRUST } \\
\hline Cover (44) & $\begin{array}{l}\text { pie }(20) \text {, bread ( } 15) \text {, pizza ( } 4) \text {, cake, } \\
\text { edge, sandwich, wound, shell }\end{array}$ \\
\hline \multicolumn{2}{|r|}{ 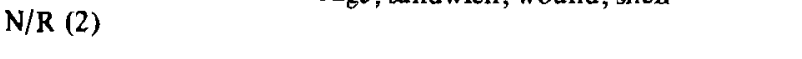 } \\
\hline \multicolumn{2}{|l|}{ DASH } \\
\hline Race (25) & $\begin{array}{l}\text { run }(15) \text {, off }(2) \text {, fast ( } 2) \text {, away, relay, } \\
\text { start, on, rush, } 100 \text { yard }\end{array}$ \\
\hline Punctuation (6) & dot (5), colons \\
\hline Dashboard (4) & board (3), car \\
\hline Small amount (3) & salt (2), pepper \\
\hline Flair (1) & gallantry \\
\hline$?(7)$ & hound (4), mash (2), dog \\
\hline \multicolumn{2}{|l|}{ DATE } \\
\hline Time (24) & $\begin{array}{l}\text { time (8), today (3), April (2), book } \\
\text { (2), month (2), "April } 1_{1} " 44-1-76, " \\
\text { calendar, numbers, year, June, "day/ } \\
\text { month" }\end{array}$ \\
\hline Appointment (14) & $\begin{array}{l}\text { girl (6), couple, dinner, guy, place, } \\
\text { male, love, bride, with }\end{array}$ \\
\hline $\begin{array}{l}\text { Fruit (6) } \\
\text { N/R (2) }\end{array}$ & nut (3), fig (2), fruit \\
\hline \multicolumn{2}{|l|}{ DECK } \\
\hline Platform (29) & $\begin{array}{l}\text { boat (10), ship (10), poop ( } 2) \text {, swim } \\
(2) \text {, surface, tennis, wood, plane, steel }\end{array}$ \\
\hline Cards (15) & cards (14), hand \\
\hline $\begin{array}{l}\text { Decorate (1) } \\
\text { N/R (1) }\end{array}$ & halls \\
\hline \multicolumn{2}{|l|}{ DESERT } \\
\hline Barren (22) & $\begin{array}{l}\text { dry (5), hot (3), sand (3), camel, bar- } \\
\text { ren, water, rat, flower, cactus, dawn, } \\
\text { Gobi, fox, arid, Sahara }\end{array}$ \\
\hline Abandon (5) & leave ( 3$)$, run, abandon \\
\hline$?(17)$ & $\begin{array}{l}\text { food ( } 5 \text {, cake }(2) \text {, ice cream (2), fat- } \\
\text { tening ( } 2) \text {, pudding, coffee, pie, now, } \\
\text { dinner, east }\end{array}$ \\
\hline \multicolumn{2}{|r|}{ - } \\
\hline \multicolumn{2}{|l|}{ DIAMOND } \\
\hline Gem (43) & $\begin{array}{l}\text { ring (15), gem (6), jewel (4), stone (2), } \\
\text { shine (2), hard (2), clear, glitters, } \\
\text { sharp, girl, tip, cutter, rough, ruby, } \\
\text { bright, love, expensive, sparkle }\end{array}$ \\
\hline $\begin{array}{l}\text { Raseball (2) } \\
?(1)\end{array}$ & $\begin{array}{l}\text { baseball (2) } \\
\text { four }\end{array}$ \\
\hline \multicolumn{2}{|l|}{ DIE } \\
\hline Death (36) & $\begin{array}{l}\text { live (13), death (8), dead (4), hard (3), } \\
\text { end ( } 2 \text {, young, nonliving, funeral, } \\
\text { grave, expire, lie }\end{array}$ \\
\hline $\begin{array}{l}\text { Mold (1) } \\
\text { Dice (1) }\end{array}$ & cut \\
\hline Dice (1) & cast \\
\hline
\end{tabular}

$?$ (6)

N/R (2)

DIGIT

Number (36)

Appendage (9)

?(1)

DIRT

Soil (44)

? (1)

$\mathrm{N} / \mathrm{R}(1)$

DIVE

Plunge (42)

Cheap bar (1)

? (2)

N/R (1)

\section{DOUGH}

Bread (37)

Money (7)

N/R (2)

DRAFT

Select (23)

Beer (18)

Preliminary writing (2)

Pull (1)

N/R (2)

DRAG

To pull (16)

Race (13)

Boring (6)

Inhale (4)

Homosexual (4)

?(3)

\section{DRAW}

Sketch (34)

Pull (9)

? (2)

N/R (1)

DRESS

Clothing (44) sheep, clothing, why, yes, do, color

number (27), calculator (2), eight, numerical, counter, phone, clock, two, unit

finger (7), toe, hand

right on

clean (5), track (4), sand (3), dust (2), dig (2), ground (2), mud (2), black (2), ball (2), brown (2), plant (2), road, organic, knees, sank, grim, dobber, rag, parasites, pay, bike, child, fun, clothes, land, filth, earth

kirt

water (13), pool (5), jump (4), swim (3), board (3), scuba (3), sky (2), in, into, tanks, deep, bell, ship, throw, boxing, cheat

tavern

yes, cup

bread (18), boy (5), nut (5), bake (3), batter, flour, play, sour, mixture, doughnut

money (6), bought

army (7), board (4), card (3), military (2), dodger (2), lottery, men, Red Sox, war, football

beer (18)

bill, draw

horse

pull (10), along, line, behind, resist, log, shuffle

race (10), city, car, chute

bore (3), dirge, on, brag

drug, joint, smoke, cigarette

queen (3), homo

snag, bag, flag

picture (10), sketch (8), write (2), art (2), paint (2), people, pencil, artist, poodle, circles, hand, paper, well, see, scribble

back (2), shoot, bridge, poker, budge, pull, horse, mister

saw, draw

clothes (7), up (6), skirt (6), maker (3), suit (3), pants (2), shirt (2), code (2), woman, clothing, belt, blouse, new, don, stockings, down, red, white, nice, 
Table 2 (Continued)

?(2)

look, fashion

mess, close

DRILL

Tool (29)

Exercise (13)

DRIP

Drops of liquid (46)

\section{DRUM}

Music instrument (42) beat (11), stick (9), hit (3), instrument

? (3)

N/R (1)

DUCK

Fowl (40)

Evasive movement (3)

? (2)

N/R (1)

DUMP

Garbage (36)

Unload (9)

?(1)

EAR

Organ (43)

\section{EXPRESS}

Rapid transport (28)

Convey (13)

Deliver (5)

FAIR

Just (18)

Carnival (15)

Light complected (6)

Average (3)

Pleasant (2)

? (1)

N/R (1) dentist, wood, down, press, electric,

class, audit, fear (2), loud (2), music (2), horn (2), bang (2), sound (2), Thoreau, major, boy, boom, bass, roll, base

bit (9), hole (7), tool (4), saw (2), oil, hammer

sergeant (5), fire (4), practice, trial, rifle, repeat

hill, me, stop, frill

drop (19), water (9), faucet (6), dry (6), coffee (3), plop, sink, filter

Display (3)

? (7)

N/R (1)

FAST

Quick (41)

Abstain (3)

? (1)

N/R (1)

soup (5), bird (5), goose (3), water (3), rubber (2), hunt (2), quack (2), feathers (2), mallard (2), hen, dinner, sitting, swan, hunting, Donald, pressed, chicken, webbed, waddle, swim, tail, lake, feet

down, under, bend

fuck, cluck

FENCE

Barrier (38)

Sport (5)

Sell (1)

N/R (2)

FIELD

garbage (14), trash (7), city (4), yard, county, all, shit, fine, smell, odor, junk, scrap, stuff, "Gandy Blvd." truck (8), unload

hump

hear (15), lobe (4), eye (4), nose (4), drum (2), ache (2), head (2), music, appendage, hole, wax, wash, cochlear, vestibular, phone, ring, pierce

train (16), fast (5), local, freeway, bus, lane, car, railroad, way

words (2), tell (2), say (2), self, opinion, show, emotions, feelings, yourself, music

pony (2), mail, check, card

just (3), even (3), right (2), game (2), equal, equitable, good, wrong, dirty, honest, foul, understanding

fun (5), grounds (3), rides (2), carnival

(2), country, Largo, circus

hair (2), lady, animal fur, light, dark

O.K. (2), teacher

day, friends

money
Cultivated land (29)

Sports arena (14)

Area of vision (1)

Area of force (1)

N/R (1)

FIGURE

Shape (35)

Calculate (8)

$N / R(3)$

FILE

Order (31)

Tool (9)

Line (4)

? (2)

FINE

Excellent (25) cool (14), blow (6), spin (3), breeze (3), blades (2), air (2), Japanese, hand, hot, turn, electric, dance, wind

club (3), mail, football, ball, letter, glamour

fare

money (6), cost (5), change (3), pay (3), train (2), taxi (2), fee (2), bus (2), price (2), ticket (2), fifty, no-frills, pass, toll, coin, token

well (2), fan

things, fair, nice, lady, honest, ideal, bare

slow (35), race, play, eagle, boyfriend, run, swift

eat (3)

brain

yard (8), gate (7), post (7), in (2), wire (2), wooden (2), link, picket, separate, sheep, jump, enclosure, repair, trap, white, protect

"engarde," duel, sword, foil, game sell

grass (5), work (3), mice (2), flower (2), stream (2), open, land, daisies, hunting, farm, park, plow, trip, crop, weeds, green, corn, wheat, plain, meadow

baseball (5), football (3), ball (2), play, goal, hockey, game

optical

magnetic

eight (5), shape (4), nice (3), girl (3), body (2), hourglass (2), good (2), picture (2), woman, skater, improvement, face, salon, drab, thin, dress, proportional, triangle, curves, skate number (2), ground (2), math, add, calculate, out

cabinet (16), papers (4), card (2), clerk (2), room, records, drawer, 13, folder, letter, look

nail (3), tooth, tool, saw, down, carpentry, cut rank (2), single, line pile, mile

good (10), okay (3), day (2), great (2), 
Penalty (11)

Delicate (8)

FIRE

Flame (41)

Shoot (1)

Terminate (1)

? (2)

N/R (1)

FIRM

Solid (35)

Company (6)

Strict (5)

FIT

Appropriate (30)

Healthy (9)

N/R (2)

FIX

Repair (33)

Drugs (8)

Mount (1)

Prearrange (1)

Stare (1)

? (2)

FLAT

Level (24)

Tire (11)

Music (3)

Apartment (2)

Paint (1)

? (5)

\section{FLEET}

Ships (39)

Swift (6)

? (1)

FLING

Toss $(31)$ arts, lousy, terrible, bind, nice, dandy, job, quality

money (4), penalty (3), court, tariff, Fun (13)

policeman, stopped

smooth (2), coarse (2), tiny, comb, ? (2)

sharp, line

now, mine

FOIL

Metal sheet (29)

hot (8), flame (4), water (4), drill (3), place (2), $\log (2)$, engine (2), alarm (2), camp (2), ball (2), boy, disaster, fighter, wind, hose, man, burn, blaze, peaceful, station

rocket

help

mire, wire

Sword (10)

Ruin (6)

?(1)

FOLD

Bend (42)

Flock (2)

? (2)

hard (15), soft (4), strong (2), grip (2), stiff (2), tight (2), grounds (2), breasts, steady, enough, loose, sturdy, solid company (5), law

strict (2), gentle, heart, offer

FOOT

Leg (44)

tight (3), clothes (3), sag (2), loose (2), together (2), room, place, pipe, out, form, dress, don't fit, perfect, humor, king, wear, college, poor, tuck, fix, pants, meet, right

seizure (2), epilepsy (2), physically able, thin, fiddle, crazy, rage

mit, bit, fun, Fla. Institute of Tech., screw

repair (10), mend (7), it (4), tool, correct, adjust, fine, right, nice, break, help, up, set, heal, car

drugs (2), heroin (2), find, dupe, needle, hit

kill

race

stare

$\operatorname{mix}(2)$

Measurement (1)

N/R (1)

FRAY

Tear (30)

Fight (7)

Nervous (2)

? (5)

N/R (2)

FRISK

Search (40)

Brisk, playful

movement (6)

FRONT

Before (44)

and (2), surface (2), smooth (2) raised, straight, hill-less, earth, foot, board, even, beach, chest, world, slope, mountains, boat, shoes, Florida, level, plain, table

tire (6), round (3), full, car

sharp (2), under

apartment, house

paint

hard, good, bat, second, out

ships (32), navy (3), boats, armada, group, gasoline

foot (2), fast (2), run, gone

meet

Facade (1)

? (1)

GAG

Choke (25)

Joke (18)

? (2)

N/R (1)

GAME

Fun (39)
(2), shot, frisbee, flung, plane, rock, hit, catch, arms

party (3), affair (2), fun (2), romance, circus, lover, bar, highland, swing nun, transform

aluminum (15), tin (6), wrap (3), paper (2), Reynolds, can, cook

fence (4), sword (3), fencing, rapier, fencer

ruin (2), again, messed up, villian, rot toil

bend (9), paper (6), crease (6), spindle (4), sheets (2), mutilate (2), open (2), towel, close, turn-over, up, fix, over, rip, out, clothes, twice, in half

flock, sheep

hold, lap

shoe (10), ball (8), toe (6), leg (3), feet (2), hand, tap, boot, mouth, print, loose, kick, walk, smell, skate, stool, guard, around, run, fall

inch

tear (5), ravel (3), jeans (2), frazzle (2), fringe (2), clothes, mess, edges, old, tablecloth, cloth, erode, torn, ragged, rope, delicate, hem, worn, tatter, string, unravel

fight (7)

frustrated, sick

horse, ray, stray, gray, play

search (15), cops (5), police (5), feel (3), check (2), touch (2), check-out, explore, rub, jail, pants, coat, pat, scratch

cat (2), run, play, blink, fast

back (34), door (2), rear (2), anterior, fence, page, porch, army, of

fake

you

choke (12), mouth (4), tie (2), stifle, reflex, shoot, strangle, quiet, sick, rule joke (11), funny (2), prank, trick, laugh, line, flop

this, flag

play (14), ball (3), Monopoly (2), fun (2), cards (2), time, chess, poker, sports, card, rules, plan, mind, soccer, Parchesi, pool, chance, bet, smile, darts, football 


$\begin{array}{ll}\begin{array}{ll}\text { Animal (4) } \\ \text { ? (2) } \\ \text { N/R (1) }\end{array} & \begin{array}{l}\text { cock (2), hunter, animals } \\ \text { chest, plane }\end{array} \\ \begin{array}{l}\text { GAS } \\ \text { Petroleum (40) }\end{array} & \begin{array}{l}\text { car (17), oil (4), fuel (2), expensive } \\ (2), \text { station (2), mileage (2), money, } \\ \text { Corvette, stamp, shortage, bill, war, up, } \\ \text { pump, strike, go, automobile }\end{array} \\ \text { Property of matter (6) } & \begin{array}{l}\text { light, liquid, chamber, heat, fart, main } \\ \text { Pand }\end{array}\end{array}$

GIN

Liquor (39)

Card game (6)

?(1)

GRACE

Prayer (20)

Poise (15)

Woman's name (9)

? (1)

N/R (1)

GRADE

Evaluative scale (41)

Incline (5)

GRATE

Rub or scrape (36)

\section{Gridiron (4)}

? (4)

N/R (2)

\section{GRAVE}

Burial place (41)

Serious (5)

GREEN

Color (39)

Immature/ inexperienced (4) ? (3)

GRILL

Cooking surface (40)

Question relentlessly light, liquid, chamber, heat, fart, main

tonic (12), drink (4), booze (3), alcohol (3), liquor (3), rum (3), drunk (2), bottle (2), mill (2), vodka, seven, dry, rickey, bathtub

Rummy (5), cards

fin

prayer (4), blessing (3), God (3), church (2), faith, good, amazing, religion, meal, gift, thanks, saved poise (4), calm, nice, gazelle, coordination, figure, smooth, women, manners, beauty, delicate, style

Slick (4), Kelley (3), name, Allen

face

"A" (17), mark (5), school (4), average (3), milk (2), improve, letter, pass, good, score, position, low, report, eeech, period

hill (2), incline (2), lever

cheese (13), grind (4), carrots (2), nerves (2), onions (2), scratch, chills, slice, sharp, cut, punish, teeth, rub, grit, irate, ear, Parmesan, corn

fence, door, iron, grill

hill, sometimes, gring, Kate

dead (15), yard (5), death (4), dig (4), stone (2), hole (2), die (2), site (2), digger (2), bury, robber, shallow bad, decision, somber, serious, stern

grass (9), blue (5), yellow (4), red (3), color (3), light (3), tree (3), gray, spade, ball, berets, jade, house, leaves, celery, money

apples, king, young, nice mean, spleen, reg.

Costume (4)

? (1)

N/R (2)

HAIL

Pay tribute (19)

Ice particles (17)

Call (7)

? (3)

HAM

Meat (42)

\section{Amateur}

radio operator (2)

Incompetent actor (2)

HAND

Appendage (41)

Cards (1)

?? Combining form (1)

? (1)

N/R (2)

HANG

Execute (21)

hamburger (7), cook (6), cheese (5), food (4), barbeque (4), steak (3), hot (2), grate, bar, toast, ground, cooker, onions, meat, eat, burner

Suspend (24) mill, dog

meat (7), teeth (3), coffee (3), bump (3), chew (2), in (2), rub, pulverize, bones, cut, flour, stone, file, grit, meal, grate, wheat, corn, churn, eat, mince, smallet work (2), routine, school, hard mead (2), find

dirt (7), hog (6), earth (4), grass (2), hard (2), air, fire, field, feet, breaking, solid, under, hay, wire, deep, land, leaf meat (2), grind (2), chuck, coffee, peanuts, beef base, work, rules figure

bad (8), smoke (4), smoking (3), practice (2), break (2), inveterate, drugs, constant, finger, bother, sameness, same, repeat, problem, strength, drinking, do, bite, dope, form, awful, fun, conditioned, pattern, habitat nun (3), riding Babbit

chief (7), king (2), Mary (2), praise (2), cheer (2), Hitler, song, queen, friend rain (7), snow (3), ice (2), sleet, weather, stone, storm, bullets wave (2), call, command, yell, taxi, hello way, harty, mail

bone (7), eggs (6), cheese (5), meat (4), food (3), pig (3), pork (3), steak (2), hog, hock, bacon, eat, sandwich, breakfast, fat, yams, lamb

radio (2)

actor, you

fingers (11), ball (5), shake (5), arm (4), foot (4), work, appendage, gun, write, over, sewn, ring, leg, good, out, rub, job

poker

some

name

dead (5), man (4), noose (3), neck (3), rope (3), kill (2), suicide

up (3), on (3), picture (3), clothes (2), out (2), stake, dangle, put up, tail, plant, high, down, cliff, in, ten, nail 
N/R (1)

HARD

Rigid (34)

Difficult (1i)

N/R (1)

HARP

Musical instrument (36) music (8), angel (6), instrument (3),

Harangue (7)

$?(1)$

N/R (2)

HATCH

Bear young

from an egg (24)

Vertical opening (17)

?(4)

N/R (1)

\section{HAUNT}

Ghost (41)

Frequented place (2)

? (3)

HEAD

Cranium (44)

Ship's bathroom (1)

N/R (1)

\section{HEAT}

Temperature (44)

? (2)

\section{HEM}

Cloth border (40)

Hesitate in speaking (5) haw (3), throat, ditto

N/R (1)

HIDE

Conceal (42)

Animal skin (4)

HOP

Small jump (44)

jump (21), skip (10), scotch (6), strings (3), heaven (2), play (2), sing (2), aeolian, soft, harpsichord, blues, melody, flute, heavenly, sound, chord, finger

Marx (2), nag (2), harping, harangue, jaw

sharp

dress (14), skirt (10), sew (9), shirt (2), line (2), darn, up, seam

Informal dance (1)

Flower (1)

HORN

Instrument (42)

Antler (2)

? (2)

HOST

Social (37)

Organism (5)

Eucharist (1)

? (2)

N/R (1)

HOUND

Dog (45)

N/R (1)

chicken, yolk, coop, hen

cover (3), open (3), boat (3), submarine (2), top, roof, door, ship, lock, back

hatchet, fast, batch, act

ghost (17), house (14), spook (3), scare (2), mansion, castle, witch, hill, frighten roost, drink

dog, seek, gaunt

hair (9), face (5), ache (4), top (3), brains (3), shoulder (3), round (2), skull (2), feet, eyes, toes, think, bald, mind, neck, ear, comb, gear, cutter, board, shop

bathroom

hot (11), cold (9), warm (5), cool (3), sun (2), sweat (2), pad, kitchen, cook, wave, Ben-Gay, liniment, stroke, fire, ray, temperature, ham, summer eat, beat

Jolt (6)

? (2)

JERK

Abrupt motion (29)

Stupid person (16)

$?(1)$

JOINT

Joining point (23)

Drugs (15)

Cheap tavern (4)

Together (2)

? (2)

seek (23), find (4), out (2), fear (2), away (2), go seek, covered, run, avoid, under, in, bushes, face, conceal

$\tan (2)$, tanning, skin

JUICE

Liquid of fruit (46)

KFRNEL

Grain (43) bunny, kangaroo, grasshopper, run, rabbit, along, rock

dance

beer

blow (22), car (4), honk (3), flute (2), trumpet (2), blower, play, sound, loud, cab, blast, French, beep, bugle

bull, broken

Bob, scorn

hostess (11), party (8), guest (8), talk, man, guard, star, maitre de, favorite, social, friend, entertainer, see parasite (2), tick, cell, America cake

ghost, most

dog (37), track (2), ground, Bluetick, Baskerville, chase, heaven, cat

jelly (23), bread (3), strawberry (2), raspberry, grape, jar, butter, toast, jell door (4), stuck (3), close, up, wreck session

ham

jelly (7), lid (6), bottle (4), glass (4), jam (4), mason (3), container (2), ball (2), pickle, mustard, jug, hold, mayonnaise, vase

open (2), shake (2), sway, bell bar (2)

off (9), pull (6), jump (3), twist (2), knee (2), twitch (2), snatch, hit, dance, shake, rope creep (2), stupid (2), fool (2), nurd (2), soda (2), idiot, bozo, you, person, shmuck, student

merk

knee (4), elbow (4), arm (2), bone (2), leg, fingers, shoulder, connection, hinge, junction, meat, lock, appendage, move, point

smoke (6), pot (4), reefer, marijuana dope, roll, water

bar (3), place

with, together

scale, event

orange (35), drink (4), fruit (3), bar (2), apple, glass

corn (42), cereal

dog, Saunders 
N/R (1)

KEY

Lock (42)

Low island (2)

Essential (1)

Piano (1)

KICK

Strike with

the foot (42)

\section{Reaction (2)}

Stimulating effect (1)

?(1)

KID

Child (29)

Young goat (10)

Hoax (6)

?(1)

KIND

Sympathetic (39)

Sort (4)

? (3)

LAND

Property (41)

Return (verb) (3)

Lord (euphemism) (1)

?(1)

LAP

Sitting (21)

Drink (4)

Dog (11)

Complete circuit (6)

Lapland (1)

? (3)

\section{LEAF}

Plant (41)

Sheet (3)

Name (1)

N/R (1)

LEAN

Thin (22)

Slant (21) lock (18), door (5), hole (3), club (3), car (2), open (2), opener, chain, turn, apartment, in, fit, room, unlock, lost Biscayne, West

word

board

ball (11), hurt (5), foot (3), hit (3), leg

(2), football, shin, karate, shoe, over, punch, boot, horse, ass, fall, pant, stomp, bull, tree, feet, punt, strike, soccer

back (2)

grass

bit

child (17), boy (2), brat (2), Cassidy, monster, pupil, sister, adult, parent, boxer, Billy

goat (9), glove

fun, toy, joke, laugh, play, wonder death

? (2)

$\mathrm{N} / \mathrm{R}(1)$

LEFT

Direction (43)

Political (2)

Remain (1)

LETTER

Postal (42)

Alphabet (2)

?(1)

N/R (1)

LIE

Falsehood (37)

Recline (9)

LIGHT

Illumination (42)

nice (11), good (3), generous (3), mean (3), pleasant (2), gentle (2), unkind, soft, gently, person, just, hard, word, cold, love, cruel, dear, humble, relative, folk, queen

type (2), different, pair

find, yes, mind

water (6), owner (4), fill (3), dirt (3), scape (3), food (2), earth (2), acre (2), mine (2), wild, open, world, expensive, plain, Tarra, plot, USA, free, development, property, sea, lover, fields

plane (2), ho

"O'Goshen"

Guthrie

sit (9), legs (4), seat (2), lady, suit, pants, thighs, girl, love

drink (2), sip, lick

ambiguous-can be in either category

run (2), around, card, swim, relay

Lander

cap, hap, wap

tree (27), green (4), maple (2), gold(en) (2), mold, oak, jump, lovely, two, rake through (2), tablet

Erikson

Legislative (11)

?(1)

LOCK

Fastener (43)

fat (6), meat (5), thin (4), beef (2), tall (2), skinny, steak, slender over (3), forward (2), bend (2), to (2),

Weight (4)

LIKE

Affection (41)

Similarity (5)

LITTER

Refuse (26)

Cat (7)

Stretcher (1)

? (3)

LOAF

Baked food (41)

Idleness (5)

LOBBY

Anteroom (34) stand, shed, post, shoulder, top, fall, against, side, on, relax, toward, railing school, mean

right (41), hand, field

radical, gauche

over

mail (12), write (8), opener (6), stamp (5), send (3), friends, white, recommendations, read, man, head, tell, arts alphabet, red bed

truth (10), cheat (7), fib (3), bad (3), detector (2), told, life, untruth, say, falsify, white, honest, machine, true, doubt, false, nope

down (4), sit, back, still, low, sex

Multiple young (9)

hotel (12), motel (3), sit (2), wait (2), hall (2), door, theater, meeting place, open, seat, Hilton, building, clerk, hospital, dorm, relax, couch, room government (3), Congress (3), Washington (2), politician, lobbyist, provoke hobby

key (15), door (13), up (3), secure (2), open (2), out, pick, chain, smith, tight, combination, seal, pad

John, mock, heed 


\section{LOG}

Wood (39)

Cumulative record (4)

Math (2)

N/R (1)

LOT

Land parcel (35)

Amount (10)

Destiny (1)

MAJOR

Significant (29)

Military rank (15)

Music (1)

? (1)

MARBLE

Type of limestone (30)

Small, hard ball (15)

N/R (1)

MATCH

Pair together (23)

Ignite (22)

$\mathrm{N} / \mathrm{R}(1)$

MEAN

Unkind (32)

Average (13)

Intend (1)

MIND

Consciousness (39)

Obey (3)

? (2)

N/R (2)

MINE

Possession (29)

Excavation (12)

Explosive device (3)

$?(2)$ cabin (16), tree (6), fire (5), wood (4), roll (3), flume, burn, logger, water, cable

diary (2), book, back

arithmetic (2)

land (13), house (3), car (3), parking (3), sale (2), plot (2), empty (2), ground, burial, ranch, rent, vacant, baseball, housing

little (3), less (2), much (2), of, bunch, money

luck

minor (18), psychology (6), most (2) important, college, problem general (5), army (3), colonel (3), captain, sergeant, Lt. colonel, rank key

major

table (9), statue (3), floor (3), stone (2), granite (2), fireplace, tile, block, hard, slab, sink, arch, grind, mantle, cake, candy

shoot (3), glass (2), play (2), game (2), round (2), colored, ball, cat's eye, blue

game (7), mix (3), maker (2), choose, colors, alike, set, together, miss, sure, mate, good, up, catch

fire (6), light (5), book (3), strike (2), box (2), stick, incendiary, cover, cigarette

nasty (10), nice (3), bad (2), mad (2), cross (2), man, hateful, hard, kind, dog, happy, machine, snake, ugly, good, wicked, word, Joe Green

average ( 8 ), mode (2), standard deviation, median, measure understand

brain (11), matter (8), think (4), game (4), control (2), head (2), boggle, cognition, intellect, soul, close, thoughts, psychology, abstract

good (2), obey

find, yes

yours (22), belonging, ours, his, my, selfish, pen, mind coal (5), gold (2), shaft (2), dig, ore, salt

field, land, sweep

fine (2)
MINT

Flavoring (27)

Manufacture (19)

MISS

Fail to hit (18)

Unmarried woman (17)

Feel loss (9)

?(2)

MOLD

Fungus (24)

Pattern (20)

$?(2)$

MUG

Container (33)

Assault (7)

Face (4)

? (2)

NET

Meshed fabric (37)

Remainder (9)

NOTE

Memo (33)

Musical tone (6)

Observe (4)

? (1)

N/R (2)

NOVEL

Book (41)

New (5)

NUT

Shelled fruit (29)

Eccentric person (8)

Hardware (7)

? (1)

N/R (1)

ORDER

Organization (19) candy (13), julep (4), flavor (2), spear, gum, cool, taste, tea, chocolate, spice, sneeze

green (8), money (5), condition (2), coin, stole, gold, fortune

hit (7), error (3), wrong, not hitting, out, shot, pass, ball, pitch, construe Ms. (3), Mrs. (3), girl (3), America (2), Mr. (2), young, lady, single, pretty you (4), school, Dru, Mary, me, absent hiss, piss

bread (8), mildew (5), slime (2), cheese (2), fungus (2), yeast (2), penicillin, green nurospora

jello (5), shape (4), clay (3), lace, iron, candle, make, round, fit, ceramic, model

hold, cold

beer (10), coffee (7), cup (5), glass (3), pottery, stein, jug, pitcher, jar, warm, drink, shaving

rob, New York, hit, stick-up, shoot, rape, wallet

shot (2), picture, face

rug, loose

tennis (11), fish (11), ball (3), basketball (2), serve (2), badminton, hair, butterfly, cord, holes, catch, game, sweat

weight (3), worth (2), left-over, two, asset, earnings

book (12), write (3), card (3), letter (3), class (2), paper (2), message (2), mark, take, pencil, exam, memo, pad music (4), musical, single important, worthy, number, this rote

book (27), read (12), chapter, best new (2), marvel, unusual, stimulus

shell (4), squirtel (4), eat (3), walnut (2), tree (2), cracker, crack pecan, fruit, food, bread, hard, almond, peanut, pea, cake, chest, cashew, Brazil house (2), crazy, weirdo, weird, square, person, dumb

bolt (7)

ball

law (4), rank (4), chaos (2), sequence (2), mess, papers, fix, file, neat, day, species 
Request (16)

Command (10)

? (1)

ORGAN

Musical instrument (29) music (10), piano (7), grinder (3), player (2), play (2), instrument, church, pipe, mouth, Hammond

Anatomy (17)

body (4), ear (3), sex (2), liver (2), heart (2), intestine, penis, lungs, kidney

PACK

Luggage (37)

Group (8)

$N / R(1)$

\section{PAGE}

Paper (40)

Servant (1)

Call for (1)

? (3)

N/R (1)

PANEL

Section of

a surface (24)

Group of people (20)

? (1)

N/R (1)

PASS

Go by (18)

Transfer (13)

Successful completion (6)

Ticket (5)

Narrow opening (2)

? (1)

N/R (1)

PAT

Gentle tap (29)

Name (14)

Exactly suitable (1)

? (1)

N/R (1)

PEER

An equal (38)

food (3), form (3), now (2), cook, more, blank, pizza, "ask for," "two burgers and a coke," number, mail command (5), court (3), demand, call truth

mule (2), camping (2), hike (2), clothes, walk, move, leave, knap, bag wolf (3), dogs (2), cigarette, beer, motorcycle

book (15), paper (5), one (4), number (4), turn (3), yellow (2), novel, read, two, news, first, nine, turner

servant

intercom

Ann, Bryant, rage

wood (7), wall (6), truck (4), display (2), wagon, dash, button, closet, room discussion (6), group (2), debate (2), question(s) (2), experts, gossip, judge, people, board, host, quiz, jury

Mantel

go (3), out (3), over (2), up (2), bridge, leave, under, by, turn, B7, "let by," ball

football (3), throw (3), forward (2), give $(2)$, touch, catch, receive

fail (4), course, class

ticket, port, weekend, permit, privilege mountain

a grille

back (5), touch (4), pet (4), tap (4), strong (2), caress (2), like, head, down, hit, scratch, fondle, hat, hug

Nixon (4), Boone (2), Zurflieh, brother, name, girl, roommate, friend, Helen, Mike

perfect

bat

group (13), friend (11), equal (4), back (17), trip (3), rat (3), horse (2), N/R (2) person (2), age, less, professional, counseling, student, level, teacher, comrade
Searching look (5)

? (3)

PEN

Writing tool (39)

Enclosed area (4)

? (2)

N/R (1)

PERCH

Sit (25)

Fish (18)

? (1)

PET

Domestic animal (38)

Fondle (4)

Brand name (3)

? (1)

Prairie (10)

? (1)

PLANE

PITCH

Throw (31)

Frequency (8)

Persuade (3)

Erect (1)

Resin (1)

? (2)

\section{PITCHER}

Container (24)

Baseball (21)

N/R (1)

PLAIN

Simple (35)

Airplane (30)

Flat surface (8)

Carpentry tool (4)

? (4)

PLOT

Piece of ground (16)

look (2), in (2), peep

beer, steer, dock

pencil (16), write (10), ink (5), knife (3), pal (2), paper (2), ball

pig (3), crib

warm, spend

bird (15), sit (9), on

fish (18)

belch

dog (24), cat (4), animal (3), store (2), food, fun, fish, mouse, mice

pat, love, feel, caress

milk (3)

peeve

throw (13), ball (9), baseball (2), hit (2), toss (2), curve, game, catch

sound (2), voice, tone, even, sing, under, high

sell, sales, line

tent

sap

hitch, ditch

glass (5), milk (4), water (4), container (3), "kool aid" (2), beer (2), glasses, sangria, drink, plant

throw (5), baseball (4), catcher (4), game (2), ball (2), mound (2), Yankee, catch

fancy (5), ordinary (4), Jane (4), simply (2), different, mousey, average, simple, uninteresting, truth, white, clothes, common, folk, blah, drab, air, complex, homely, custard, bare, neat, clear, exceptional

flat (2), plane, mountain, old, field, Indian, desert, grass, straight chain

fly (11), air (9), sky (2), airport, takeoff, propeller, land, ticket, trip, crash, jet

straight, level, horizon, surface, geometry, point, area, smooth shave, cut, carpentry, sand

fancy, dress, simple, Indians

Conspiracy (13)

Story (9) ground (6), land (5), cemetery (2), garden, soil, corn

plan (6), kill (3), steal, assassination, destroy, trick

story (5), subplot, novel, movie, thickens 
Graph (7)

N/R (1)

graph (5), course, dots

PRESS

\section{POACH}

Cooking (27)

Hunt illegally (18)

?(1)

POKER

Card game (43)

Metal rod (3)

POOL

Swimming (32)

Billiards (13)

?(1)

PORT

Harbor (30)

Ship's side (9)

Beverage (6)

? (1)

POST

Mail (25)

Pillar (13)

Following (5)

Base (1)

? (2)

POT

Marijuana (24)

Container (20)

\section{POUND}

Unit of weight (25)

Hammer (13)

Livestock enclosure (5) Monetary unit (2)

N/R (1)

PRESENT

Gift (21)

Current (15)

At hand (8)

? (1)

N/R (1) egg (26), boil

steal (13), hunt (2) take, possum, kangaroo

coach

chips (14), cards (9), game (8), face (4), hand (2), player (2), jack, ante, joker, stake

fire (2), fireplace

swim (19), water (7), cool (2), slide, sun, party, swimmer

cue (3), hall (3), table (3), play, room, game, billiards

ear

ship (10), boat (3), Tampa (2), Everglades (2), harbor (2), authority, entry, Arthur, of, sea, o'call, water, sailor, cove, air, voyage

starboard (5), right, side, hole, left wine (4), brandy, tawny court

office (15), mail (4), man (3), master, letter, bonds

fence (3), hole (2), lean, pole, nail, column, display, whippin', pillar, time mortem (2), after, pre, operation military

hast, ghost

smoke (10), "grass" (5), marijuana (3), dope (2), flower, joint, plant, bad pan (4), stew (3), hot (2), cook (2), gold, soup, tea, stove, kettle, cooking, dirt, belly, utensil

spot, dot

cake (5), ounce (5), weight (4), meat, grass, heavy, sugar, loaf, water, hamburger, ham, amount, pint, lb.

hit (4), beat (2), thump, out, nail, hammer, desk, bang, hound

(5) Male sheep (32)

England, shilling

gift (13), Christmas (4), receive, box, give, birthday

past (6), now (5), future (2), last, moment

absent (5), here (3)

Nixon
Strike (14)

Compress (26)

Printing (20)

PRUNE

Dried fruit (37)

Cut (6)

? (2)

N/R (1)

PUNCH

Strike (25)

Drink (20)

$?(1)$

PUPIL

Student (26)

Eye (20)

QUACK

Sound (27)

Charlatan (18)

N/R (1)

QUEEN

Royalty (39)

?(1)

$\mathrm{N} / \mathrm{R}(1)$

QUIVER

Arrow case (13)

Sport (41)

Noise (3)

Illicit business (1)

? (1)

RAM

RANGE

Cooking stove (18)

Horizontal

distance (15)

Open area (12)
Homosexual (5)

Tremble (33)

\section{RACKET}

iron (10), hard (4), push (2), pull (2), compress, shirt, squash, dress, mash, against, leaf, button

newspaper (5), news (5), printing (4), reporter, book, paper, journal, agent, stop

juice (10), fruit (7), wrinkled (5), pit (3), face (2), sauce, cocktail, Danish, eat, dried, constipated, plum, raisin, crap, shit

trees (3), cut, trim, pick

swoon, cold

hit (9), Judy (4), nose (3), out (2), first, job, slug, card, mouth, bag, hurt bowl (4), drink (4), fruit (2), Hawaiian (2), planters, ball, juice, drunk, apple, vodka, party, wet bunch

student (19), school (3), me (2), teacher, study

eye (20)

duck (26), clack

doctor (16), charlatan, physician

king (27), bee (5), Mary (2), aristocrat closet, drag, fairy, gay, queer Ellery

shake (22), cold (3), shudder (2), scared, palpitate, afraid, shiver, tremble, lips

arrow (9), bow (2), silver (2)

tennis (26), ball (9), club (6)

noise (3)

gambling

bracket

sheep (9), goat (6), horn (3), football (2), ewe (2), lamb, bull, Los Angeles, Pan, butt, April, Leo, Aries, ham, hill shove, push, hit, smash, damn, rod, jet, thrust, rage, pass, on, goal, jam, ocean

oven (9), stove (8), hot

width (2), statistics (2), high (2), spread (2), wide, reach, finder, altitude, distance, intensity, meters golf (3), home, space, horses, war, fire, 
N/R (1)

RANK

Row/sequence (37)

Offensive (6)

? (2)

N/R (1)

RARE

Scarce (31)

Incompletely

cooked (15)

RARE

Scarce (33)

Incompletely

cooked (10)

? (2)

N/R (1)

RASH

Skin irritation (42)

Reckless (4)

\section{RECORD}

Phonograph (32)

Account of events (8)

N/R (6)

\section{REEL}

Spool (43)

Dance (1)

Swaying motion (1)

N/R (1)

RELISH

Pickles (36)

Savor (10)

REST

Repose (34) missile, ranch, pasture, rifle

Remainder (0)

? (12)

order (9), file (5), army (2), place (2), list (2), top, colonel, check, chief, serial, military, soldier, title, level, low, age, vacation, position, money, priority, first, high

stink (2), odoriferous, smell, spoiled, rude

hank, steel

RIB

Curved support (42)

Tease (2)

N/R (2)

RIDDLE

Conundrum (39)

unusual (6), diamonds (2), bird (2), unique (2), valuable (2), earth (2), few (2), scarce, gem, divine, book, find, expensive, coin, seldom, common, abundant, novel, case, bit

steak (6), medium (4), meat (3), welldone, hare

unusual (5), unique (4), coin (3), earth (2), common (2), diamond, love, exotic, bird, book, find, antique, occasion, few, gem, expensive, abundant, novel, plenty, UFO, redundant, money steak (4), meat (2), well-done, medium, hare, bit

back, fare

red (6), heat (6), itch (6), measles (4), hive (3), skin (3), bumps (2), poison (2), diaper, allergy, sore, redden, irritation, break, break-out, scratch, leg, " 2 ",

brash (2), harsh, hasty

player (9), music (5), play (3), tape (2), album (2), listen, phonograph, changer, label, shop, store, table, sound, Bob Dylan, jockey, dance maker, everything, write, talk, G.P.A., top, world, on

rod (15), fish (11), fishing (10), in (3), movie, recorder, roll, tape

Virginia

train

Perforate (1)

? (4)

N/R (2)

RIGHT

Correct (24)

Direction (22)

RING

Circular object (28)

Sound (16)

Group (1)

N/R (1)

ROAD

Path (45)

N/R (1)

ROCK

Stone (29)

Music type (15)

?(1)

N/R (1)

ROLL

Tumble (23)

Bread (12)

Reverberating sound (7)

Listing (2)

pickle(s) (12), hot dog (11), tray (2), garnish (2), mustard (2), radish, dish, hot, hamburger, vegetable, food, jam enjoy (3), like (2), me, savor, you, love, treasure

? (1)

N/R (1)

ROOM

Space (44) wait, recuperate, nap, worst, bed, relaxation, heaven, work

bird (8), egg (2), offspring, west

Adam (9), cage (7), steak (4), eye (3) chest (3), bone (3), Bar-B-Q (3), eat, spire, pork, break, slab, meat, heart, chew, cracked, roast

rub, joke

joke (15), puzzle (8), thyme (4), question (3), game (3), solve (2), truth, this, me, poem

holes

Nelson, fiddle, none, charm

wrong (22), on (2)

left $(20)$, hand, arm

finger (7), diamond (6), round (3), wedding (2), circle (2), circus (2), around, marry, opal, gold, jewelry, bought

bell (11), bicycle, sound, loud, rang, ding

leader

runner (6), street (5), drive (5), travel (4), sign (3), dirt (2), car (2), hug, asphalt, shell, narrow, barrier, wind, track, pavement, to Rio, work, curves, go, test, run, SR 581, map, highway

stone (5), hard (5), heavy (2), pile, big, garden, ages, boulder, hammer, gem, sand, pebble, shell, bottle, salt, pet, minerals, river, throw, hunter roll (7), music (5), market, dance, star sock

down (3), over (3), $\log (2)$, tide, turn, stop, ball, dirt, joint, out, play, dog, a-coaster, on, tumble, hill, along, dice butter (4), bread (4), eat, bun, food, dinner

rock (5), pitch, stomach

call (2)

hold

class (8), house (4), bed (3), bath (2), space (2), door (2), live (2), hall (2), crowded, cold, small, large, apartment, 
N/R (2)

\section{ROOT}

Base (41)

Rummage (1)

? (2)

N/R (2)

ROSE

Plant (44)

Rise (2)

ROW

Propel (27)

Straight line (18)

Quarrel (1)

RULER

Measurement (36)

Govern (9)

? (1)

RUNNER

Race (44)

Narrow covering (1)

N/R (1)

SACK

Bag (43)

Bed (3)

SAP

Fluid (38)

Stupid person (4)

?(1)

$\mathbf{N} / \mathbf{R}(3)$

\section{SAW}

Cutting tool (31)

Observed (12)

Proverb (1)

? (2) curtain, mirrors, dorm, ball, place, mate, two, freshen, board, day, empty, study, sleep, place

tree (12), beer (7), plant (6), potatoes (2), evil (2), square, tall, bottom, legume, doctor, stem, ground, hair, tap, word, earth, source

pig

hoot, shoot

flower (19), red (9), bush (4), smell (3), petal, plant, hips, queen, daisy, thorn, wine, bud, sweet

hop, stein

boat (23), oars (2), fish, paddle

column (6), field (2), hoe, series, aisle, seat, seven, two, 3rd, line, sew, seats angry

SCALE

Measure (39)

Climb (2)

Epidermis (4)

?(1)

SCRAP

Fragment (41)

Quarrel (3)

? (1)

N/R (1)

SCREEN

Partition (32)

Project (12)

? (1)

N/R (1)

SEAL

measure (21), twelve (2), number (2), foot (2), inches, slide rule, staple, geometry, pencil, stat, yardstick, desk, length

king (7), emperor, kingdom

none

Animal (23)

Close (14)

Emblem (5)

? (3)

track (5), road (5), fast (4), distance (3), mile (2), race (2), up (2), athlete (2), sprint (2), walker, mail, ran, person, mark, grass, slow, base, quarterback, jogger, swimmer, run, message marathon, football, gun, miler ng

N/R (1)

Time (25)

Ordinal position (21)

SET

Place (26)

potato (12), race (5), bag (5), back (3), burlap (2), grain (2), cloth (2), lunch (2), nap, stack, loose, brown, wine, flour, tie, hold, up, mill

bed (2), time

tree (23), pine (2), maples, spring, "life-giving liquid," "maple syrup," resin, strength, blood, pitch, rubber, life, sip, syrup, plants

sucker (2), POW, dope

rap

hammer (6), cut (6), wood (6), horse (3), tool (2), chain (2), carpenter, stool, shop, sharp. blade, rip

see (9), came, eye, seen

adage

buck, order

Collection (11)

Receiver (6)

? (2)

N/R (1)

SHARE

Portion (44)

Plow (1)

N/R (1)

SHARP

Pointed (40)
SECOND

weigh (14), weight (12), measure (3), down (2), music (2), judge, hundred, inch, sing, musical, note

climb (2)

fish (3), skin

Hale

iron (6), junk (6), paper (5), meat (4), metal (3), book (3), waste (3), sew, extra, grease, trash, dog, garbage, city, food, piece, left-over, crap

fight (3)

snap

window (8), door (7), porch (4), bug (3), out (2), cover (2), wire, play, net, silk, gem, flies

movie (5), film (3), printing, print, projector, test

preen

animal (6), lion (3), ball (2), water (2), swim, bark, fish, black, seaquarium, mammal, sea, cold, clap, sea lion close (4), letter (2), lick (2), open, protect, it, kiss, wrap, tight

corporate, Easter, approval, Florida, affix

peal, start, take

minute (12), time (6), short (2), tick, millisecond, split, less, one

first (8), third (4), chance (2), place (2), guess, syllable, best, hand, helping

up (4), hair (3), down (3), place (3), back (2), sit (2), hen, pain, put-on, rigid, close, on, table, screw, get collection (2), group (2), theory, match, math, chess, tennis, china, decision

TV (6)

met, still

give (7), alike (3), stock (3), cropper (3), food (2), crop (2), fair (2), equal (2), money (2), generous, cookies, children, split, toys, "give and take," part, holders, help, land, parcel, trust, kind, lend, friendship, bread, bonds, yes equipment 
Clever (3)

Pungent (2)

?(1)

SHED

Throw off (25)

Shelter (19)

?(1)

N/R (1)

SHELI

Outer covering (41)

Explosive (3)

?(1)

N/R (1)

SHIFT

Change position (30)

Work period (8)

Chemise (7)

Trick (1)

SHIP

Boat (43)

$?(2)$

N/R (1)

SHOOT

Discharge (45)

? (1)

SHOT

Discharge (26)

Medical (7)

Liquor (6)

Athletic (3)

Attempt (3)

Ruined (1)

SHOWER

Bathe (40)

Light rain (6)

SIGN

Symbol (29)
(4), blunt (2), cut (2), pencil (2), soft, axe, stick, object, edge, point keen (3)

cheese, awful

harp

Signature (17)

SINK

skin (5), hair (4), remove (2), light (2), went, lose, leave, feathers, grace, coat, hold, fell, drop, "get out of," lawn, birds

tool (3), barn (3), storage (2), wood (2), hut (2), lean-to, house, cow, backyard, horse, building, store yes

Basin (26)

Descend (20)

SKIRT

Garment (39)

Border (4)

? (3)

sea (10), beach (8), fish (5), oil (5) feet, macaroni, game, crab, egg, sand, outside, road, outer, rock, clam, collection, hard

mortar, casing, shock

constant

SLAB

Flat, thick piece (42)

? (3)

change (11), gear (9), car (3), move (2), alter (2), rearrange, sands, less work (5), job, third, graveyard dress (3), skirt (2), slip, clothes cheater

N/R (1)

SLIDE

Slip (37)

(Verb)

boat (10), sail (9), water (4), vessel (3), sea (2), ocean (2), port (2), shore (2), shape, big, carrier, dock, fleet, ahoy, mast, fools, tree

flip, shop

Image plate (9)

SLING

Weapon (34)

Supporter (11)

gun (13), kill (6), bird (3), out (2), bull (2), fire (2), bang (2), down (2), retrieve, sport, hunt, bear, over, star, turkey, skeet, dice, hit, ducks, pool, buffalo

hoot

gun (15), kill (3), hurt (2), fired, far, at, sheriff, hit, bullet arm (2), blood, allergy, helpful, rabies, injection

whiskey (4), jigger, bourbon

put, sling, discus

dark (3)

worn

N/R (1)

SLIP

Slide (32)

Clothes (11)

Specialized paper (3)

SLUG

Hit (20)

Mollusk (12)

Bullet (11)

Liquor (1)

N/R (2)

clean (7), water (6), bath (5), wash (4), stall (3), curtain (2), soap (2), wet (2), powder, off, head, often, body, nice, two, nozzle, tub

rain (3), cool, flower, April

SOCK

Garment (39)

Strike (6)

N/R (1)

SOLE

stop (6), neon (3), road (3), language
(2), signal, information, hands, detour, street, stimuli, traffic, post, negative, read, Aries, see, highway, omen, painter name (8), signature (2), line (2), up, autograph, document, write, paper

kitchen (8), dishes (8), wash (4), soap (2), leak, bathroom, drink, cleaner fall (3), ship (3), water (2), down (2), boat (2), swim (2), Bismark, well, hold, float, line, under

dress (15), hem (4), short (4), blouse (3), clothing (3), pants (2), slip (2), full, mini, passe, wear, slacks, legs around, table, edge, control

skirt (3)

concrete (11), stone (4), meat (3), rock (3), marble (3), morgue (2), cement (2), rib (2), granite (2), body, tomb stone, skate, piece, plot, grave, cold, flat, paint, metal

crab, blab, lab

slip (10), rule (6), down (4), door (3), fall (2), sled, move, easy, under, children, home run, snow, safe, roll, playground, board, glide

projector (3), picture (2), microscope, camera, show, film

shot (20), arrow (4), throw (3), shoot (2), fly, rubber, fling, hit, mud arm (4), cast (3), broken (2), injury, rope

fall (14), slide (7), on, bath, banana, knot, sink, slop, down, slap, mistake cover (2), undergarment (2), clothes (2), lingerie, show, skirt, shop, miss paper (3)

hit (12), boxer, fight, first, punch, batter, mug, swing, hug

worm (4), snail (3), "ugh," rain, garden, bug, hate

bullet (8), gun, shot, plug whiskey

shoe (20), foot (12), feet (3), white, Christmas, hold, food hit (3), punch, it, jaw 
Table 2 (Continued)

Fish (12)

Single (3)

? (3)

N/R (1)

\section{SOW}

Female pig (24)

Spread (verb) (19)

? (2)

N/R (1)

SPADE

Shovel (22)

Card suit (22)

?(1)

$\mathrm{N} / \mathrm{R}(1)$

SPEAKER

Speech maker (28)

Musical (15)

?(2)

N/R (1)

\section{SPELI}

Orthography (37)

Supernatural event (8)

N/R (1)

SPREAD

Diffusion (36)

Cloth cover (5)

Ranch (3)

? (1)

N/R (1)

SPRING

Season (27)

Coil (15)

Water source (2)

?(1)

N/R (1)

SQUARE

Plane figure (39)

Personality (3)

Root (3)

N/R (1) fish (10), filet (2)

single, alone, only

soul, fool, sin

pig (18), cow (4), fat (2)

reap (8), farm (4), seed (3), plant, hoe, oats, plow

low, cloth

shovel (14), dig (2), hoe (2), garden, tool, sand, dirt

black (7), cards (6), ace (4), club (2), diamond, heart, nigger

made

lecturer (4), guest (4), listener (3), teacher (3), house (2), talk (2), voice, stage, bore, orator, listen, lecture, topic, talker, podium, McCormick stereo (6), loud (2), hi-fi, acoustic, sound, noise, wires, cone, big

no, meeker

words (15), correctly (7), bee (4), English (2), write (2), number, out, error, I, game, school, well

bound (2), bad (2), cast (2), witch, trance

butter (15), jam (4), bread (4), legs (2), smooth (2), sandwich (2), pour, eagle, wise, mustard, dip, out, Playboy bed (4), satin

Texas, soil, ranch

Fred

summer (6), flowers (4), winter (2), fall (2), training (2), autumn, pretty, leaves, beautiful, time, now, break, bean, trees, enthusiasm, fresh board (3), bounce (2), bed (2), mattress (2), sprung, wire, tense, leap, jump, open

water, well

string

round (16), circle (7), dance (3), rectangle (2), shape, Latin, cylinder, blocks, city, box, triangle, inch, four, deal, table

people (2), parent

root (3)
STABLE

Shelter (36)

Secure (8)

? (1)

N/R (1)

STAFF

Specialized group (31) faculty (6), meeting (5), member (5),

Pole (10)

Music (2)

? (2)

N/R (1)

STAGE

Platform (38)

Vehicle (6)

? (1)

N/R (1)

\section{STAKE}

Post (38)

Property (2)

? (6)

STALK

Pursue (31)

Plant stem (13)

? (2)

STALL

Compartment (32)

Delay (12)

? (1)

N/R (1)

STAMP

Postage (38)

Stomp (8)

STAND

Position (40)

Structure (6)

STAPLE

Binder (29) group (2), car (2), sergeant, colleagues, workers, work, doctor, helpers, secretaries, professor, chief, room, teachers

stick (3), rod (2), cane, flag, pole, Moses, life

music, line

lane (2)

horse (29), barn (2), animals, cow, straw, stall, smell irrational, able

cheer

fright (6), actor (6), act (5), door (4) play (4), acting, platform, writer, show, stop, "Act I," game, theatre, production, curtain, crew, hand, perform coach (6)

rage

ground (4), Dracula (4), out (3), stick (3), wood (3), vampire (2), post (2), wooden (2), pole (2), heart (2), hang, nail, board, kill, burn, tent, peg, tomato, throat, lots, make

bet, claim

vane, eat, take, steak, shake, beef

hunt (12), prey (6), follow (2), catch (2), walk, pursue, leopard, stealth, chase, tiger, plot, lion, shoot

corn (6), celery (3), asparagus, tree, forest, sheath

balk, filet

horse (25), farm, garbage, "small area," shower, barn, hall, bathroom wait (3), go, stop, delay, hold, plane, car, choke, bluff, "put-off" all

letter (7), mail (7), postage (6), lick (5), collector (2), collection (2), collect, need, envelope, expensive, gas, paper, affix, post, duck down (2), out, on, rubber, money, shoe, approval

sit (17), up (9), by (3), fall (2), erect (2), upright (2), for, out, tall, feet, firm tire, ice cream, set, music, night, desk

gun (15), paper (4), together (3), page 
Table 2 (Continued)

(2), attach, machine, clip, nail, fasten

Chief commodity (12) food (5), bread (2), goods, flour, diet, sugar, maple

? (4)

N/R (1)

horse, room, singers, bird

STAR

Heavenly body (40)

Prominent

performer (6)

STATE

Territory (37)

Status (7)

Say (2)

STEEP

Incline (42)

Brew (2)

? (2)

STEER

Ox (34)

Direct (10)

$\mathrm{N} / \mathrm{R}(2)$

STEW

Food (46)

STICK

$\operatorname{Rod}(30)$

Adhesive (11)

Jab (5)

STILL

Stationary (34)

Distillery (9)

? (2)

N/R (1)

STIR

Agitate (45)

sky (10), bright (5), heavens (3), trek (3), moon (2), planet (2), shine (2), Arcturus, wish, dark, nova, galaxy, sun, David, north, "Big Dipper," spangled, system, far, Orion

piano, born, player, quarterback, music, struck

Florida (12), fair (6), union (3), city (2), country (2), Texas, New Orleans, Michigan, highway, government, bird, Indiana, Kansas, Ohio, Oklahoma, Tennessee, federal

condition (2), square, affair, be, "of mind," consciousness

case, tell

hill (11), incline (6), high (5), slope (4), climb (3), mountain (2), sharp (2), steps, tall, narrow, heep, grade, church, steeple, cliff, pit

brew, tea

throat, keep

cow (15), beef (5), cattle (5), bull (4), food, calf, horns, cowboy, castrate car (4), drive (2), wheel, lead, guide, away

beef (22), food (6), meat (4), pot (3), potato (2), vegetable (2), fish, cook, Irish, meatballs, eat, carrots, hash

ball (5), shift (4), wood (4), branch (2), cane (2), dog, stone, stir, tree, limb, club, pin, guns, throw, drum, fence, man, dip

gum (4), stuck, slip, gooie, mud, on, good, tire

kick, punch, hit, up, poke

quiet (7), water (6), calm (3), dead (2), active (2), life (2), hard, unmoving, silent, house, same, fast, here, bound, shallow, agitated, listless, mountain whiskey (4), booze (3), liquor, mill Stephen, Nash

mix (16), coffee (5), pot (3), move (3)
Prison (1)

glass, water, candy, swizzle stick crazy

STOCK

Supply (36)

Support (5)

Original (4)

? (1)

STORE

Market (33)

Elevation (2)

N/R (1)

STRAIN

Effort (30)

Filter (12)

Ancestry (4)

STRAW

Grain (30)

Worthless (7)

Drink (5)

? (3)

N/R (1)

STRIKE

Hit (34)

Stop work (10)

N/R (2)

STRIP

Undress (26)

Narrow piece (20)

SUIT

Clothing (45) spoon (2), up (2), fry, bubble, fudge, drink, burn, rotate, cook, boil, soup, market (9), bonds (5), supply (3), exchange (2), holder (2), boy (2), money (2), cows (2), fix up, business, beef, animals, merchandise, extra, item, take, room

barrel (3), block, shoe

car (2), bred, first

Jamie

grocery (6), buy (6), keeper (5), food (4), bought (2), A \& P, owner, 7.11, Maas Brothers, market, country, watch, general, open, merchandise house (4), room (3), money (2), save (2), in, more

book (11), tale (7), teller (5), tell (4), novel (2), read (2), fable (2), fairy, short, child, writer, joke, moral, bad, good, write, telling

building, floors

stress (6), work (3), try (2), brain (2), muscle (2), hard (2), back (2), lift, sprain, hardship, tax, hear, struggle, stretch, effort, labor, train, mental food (2), honey, screen, sift, straw, drink, noodles, juice, mesh, tea, water type, virus, bacteria, culture

hat (18), hay (5), stable, yellow, hay stack, bedding, floor, turkey, horse last (3), man (2), dog, testy

drink (4), sip

berry (2), stretch

hit (19), out (4), ball (2), hard (2), three (2), one, blow, down, spare, violence

union (3), picket (2), wages, hike, "on strike," Busch, work

naked (3), joint (2), tease (2), undress (2), clothes (2), disrobe (2), off (2), poker (2), nude, now, see, take-off, peel, down, pull-apart, bare, mine piece (2), bacon (2), steak (2), tear (2), land (2), enamel, wood, zebra, line, thin, sirloin, comic, stripe, door, film

clothes (12), tie (6), dress (5), shirt (3), case (3), conservative, neat, man, sweat, tux, fit, skirt, coat, up, wear, 
$?(1)$

SWALLOW

Ingest (30)

Bird (15)

N/R (1)

SWAMP

Marsh (40)

Overwhelm (5)

N/R (1)

SWITCH

Device (29)

Transference (10)

Twig (5)

?(1)

$N / R(1)$

TAB

Drink (21)

Charges (10)

Flap (7)

Tabulator (2)

Name (3)

?(1)

N/R (2)

TAG

Label (36)

Touch (10)

TAP

Strike lightly (27)

Spigot (15)

Borrow (1)

? (1)

N/R (2)

TAX

Fee (45)

?(1)

TEND

Look after (28) brown, swim, best, jacket, dirty, Inclination (14) office

shoot

throat (7), water (5), gulp (4), fish (2), eat (2), choke, chew, milk, up, mouth, digest, hard, spittle

bird (14), tail

? (2)

$\mathrm{N} / \mathrm{R}(2)$

TERMINAL

End (45)

water (6), alligator (5), mud (4), Everglades (4), bog (2), muck (2), dismal, murky, gators, hunt, fox, fever, mush, buggy, marsh, messy, foggy, mosquito, lagoon, bugs, watery, yearling shake (2), work, canoe, overload

light (12), blade (6), on (4), TV (2), off (2), board (2), train change (6), turn, turnabout, trade, over hit, punish, swat, stick, me band

Electrical (1)

TICK

Sound (24)

Parasite (19)

Movement (1)

? (1)

N/R (1)

TIE

Knot (45)

Draw (1)

drink (9), coke (7), soda (3), can, diet bill (4), check (2), tablet, ticket, leave, credit

pull (3), paper, file, beer, bit

typewriter, type

Hunter (3)

slap

TIP

Edge (26)

Gratuity (14)

Advise (5)

N/R (1)

TIRE

license (5), car (5), dog (4), mark (3), name (3), number (2), it (2), identify (2), auto, label, deer, Xmas, shirt, cloth, collar, shoes, file, plate

game (3), team (2), play, children, along, catch, touch

Automobile (41)

To fatigue (5)

TOAST

Bread (41)

dance (8), hit (3), touch (3), back (2), fingers (2), drum, thump, pat, shoulder, tip, pencil, desk, strike, rap

water (8), beer (3), sink, spring, keg, pub

out

clothes

Drink to (4)

N/R (1)

TOOL

Instrument (46)

income (8), money (7), collector (5), pay (3), man (3), government (3), cut (3), evasion (2), bite, deduction, penalty, credit, sales, dollar, cost, return, number, shelter, break

ax

TRACE

Follow (19)

Copy (19) lean (4), spend (2), toward, trend, likely, habit, move, direction, think, inclination

lend, tent

end (12), train (7), cancer (6), bus (4), airport (3), illness (3), death (2), goal, man, stop, course, fly, computer, final, last

wire

tock (10), clock (6), tac(k) (4), sound, hear, time, click

flea (7), $\operatorname{dog}(5)$, bug (2), mite (2), insect, mad, off

superstitious

Dick

know (8), bow (7), shirt (6), suit (3), neck (4), shoe (2), coat (2), dye, formal, thread, blue, hold, clip, cord, ribbon, knob, down, wife, clothes, tack win

top (7), end (4), hat (3), toe (3), turn (2), pool cue, dump, pin, pen, glass, flip, touch

money (5), waitress (2), waiter (2), pay, $15 \%$, bar, maid, restaurant point (2), off (2), hint

car (11), flat (8), iron (4), wheel (3), care (2), mark (2), blowout, Goodyear, rubber, tube, truck, bald, radial, spin, wear, tool, waist

easily (2), rejuvenate, fatigue, fire

bread (14), butter (5), breakfast (5), burnt (3), jam (3), toaster (2), cook (2), brown (2), coffee, milk, cinammon, jelly, roast

drink (3), town

hammer (9), shed (6), dye (3), work (3), instrument (2), wrench (2), kit (2), screwdriver, object, mend, box, hardware, good, rod, rock, shop, utensil, calculator, rake, awl, wheel, leather, fix, fool, saw, artifact

follow (3), trail (2), find (2), track (2), shooting, check, fire, out, trackdown, see, police, path, evidence, elementary outline (4), draw (3), picture (2), paper (2), line (2), cheat (2), copy, over, face, finger care (6), watch (4), do (4), cows (3), show (2), to (2), ignore, to go, cattle, attend, mind, give, mend 
Minute quantity (6)

$\mathrm{N} / \mathrm{R}(2)$

TRADE

Exchange (33)

Commerce (9)

Craft (4)

TRAIN

Railroad (37)

Instruct (7)

TRUNK

Luggage (27)

Main axis (9)

Prehensile proboscis (6) elephant (6)

Pants (1)

?(3)

TRUST

Faith (30)

Property (12)

$?(2)$

$\mathrm{N} / \mathrm{R}(2)$

TYPE

Print (34)

Illk (12)

VAIN

Conceit (28)

Futile (6)

?(11)

N/R (1)

VAULT

Chamber (36)

Jump (10) chemical travel, name organism

rain, Jordan

swim

kick, knife elite, bell small, bit, memory, speck, element,

exchange (6), barter (3), post (2), winds (2), off (2), give (2), made (2), baseball, wampum, turn, stamps, in, even, swap, sell, corner, share, stock, deal, draft, yes

mark (4), business, market, commerce,

union (3), work

tracks (7), station (6), engine (5), whistle (3), car (3), stop, toy, coal, choochoo, ride, travel, boat, plane, truck, freight, Europe, caboose, strain teach (2), dog, learn, sun, thought,

car (6), box (6), suitcase (3), lid (2), traveling (2), clothes, luggage, locker, large, case, Saratoga, store, key tree (8), chest

skunk, drunk, bunk

me (3), faith (3), truth (2), God (2), credible, believe, blind, mistrust, distrust, caring, obey, people, friend, loyal, gamble, honesty, judgment, like, togetherness, judge, nobody, love, must, rely upon

fund (5), bank (3), money (2), company, account

writer (12), write (5), print (4), error (4), letter (3), typist, ink, no, paper,

kind (5), blood, good, B-, style, person, crossmatch, file

conceited (5), proud (2), mirror (2), ego (2), self (2), showy, beauty, haughty, alright, stuck up, peacock, sickening, you, skin, you're, success, pretty, fool, modest, glorious

useless, hostile, allow, hope, in, futile arteries (2), weather (2), leg, shrimp, bain, blood, arm, gold, refrain

safe (17), bank (11), money (2), key, chest, coffin, box, safety, lock pole (4), fault (2), jump (2), over, leap
VESSEL

Ship (28)

Vase (14)

Hollow tube (3)

?(1)

\section{WAKE}

Sleep (24)

Wave (9)

Funeral (9)

? (3)

N/R (1)

WASH

Cleanse (46)

WATCH

Chronometer (28)

Observe (15)

?(1)

N/R (2)

WAX

Paraffin (39)

Gradually grow (?)

WEAR

Habitually carry (36)

Erode (8)

? (1)

$\mathrm{N} / \mathrm{R}$ (1)

WELL

Underground

source (26)

Positive state (20)

WIND

Air (42)

To turn (4)

WORK

Effort (35)

Employment (8)

? (2)

N/R (1) ship (22), boat (5), navy

container (5), vase (2), drinking, cup, tub, urn, carry, glass, contain

blood (3)

tressel

sleep (8), up (8), morning (5), asleep (2), arise

ship (2), slip, flood, boat, ocean, surf, water, wave

funeral (5), death (2), dead (2)

fake (2), forest

clean (10), hands (4), dry (4), clothes (4), feet (2), up (2), dish (2), scrub (2), car (2), cloth (2), rag, cleanse, wear, face, self, basin, tub, soap, body, sink, hair, shower

time (17), wrist (4), clock (2), hand (2), maker, gold, fob

watchdog (4), look (3), see (2), tower, out, observe, duty, me, listen

chair

floor (8), car (5), bees (3), shine (3), museum (2), slippery (2), candle (2), paper (2), shiny, polish, surfboard, board, figure, cleaner, sap, beauty, bar, slide, ears, skis wane (7)

clothes (16), wash (5), dress (4), pants (2), apparel, top, slip, ring, jeans, shoe, don, under, loose

tear (4), out (2), ragged, off

bear

water (18), deep (3), wishing, oil, dig, dry, field

sick (7), done (3), good (3), fine (2), get, wishes, dead, happy, trained

blow (15), breeze (4), sail (3), mill (2), north (2), rain (2), storm (2), cosmic, willows, beach, push, bag, soft, window, smooth, howl, song, cold, fall clock (3), up

hard (10), play (10), loaf, relax, horse, through, toil, ethic, difficult, easy, detail, lazy, rest, bad, run, tired, labor job (4), money, shirt, days, employed hard, cork 


\section{YARN}

Thread (34)

Fable (11)

? (1)

YELLOW

Color (44) knit (10), thread (7), string (4), wool (4), ball (2), rugs, darn, cloth, booties, spool, weave, skein tale (6), story (2), farm (2), fable danger

YIELD

Succumb (46)
(2), brown (2), flower, warm, dog, bird, journalism, fever, pad, paper, brick, sub chicken, back

give (12), stop (11), sign (5), right (4), give-in (2), slow (2), go (2), traffic, bend, allow, break, way, overcome, give-away, succumb

\section{REFERENCES}

The American Heritage Dictionary of the English Language. New York: American Heritage, 1973.

Cramer, P. A study of homographs. In L. Postman \& G. Keppel (Eds.), Norms of word association. New York: Academic Press, 1970.

Geis, M. F., \& Winograd, E. Norms of semantic encoding variability for fifty homographs. Bulletin of the Psychonomic Society, 1974, 3, 429-431.

Kausler, D. H., \& Kol.lasch, S. F. Word associations to homographs. Journal of Verbal Learning and Verbal Behavior, 1970, 9, 444-449.

Nelson, D. L., Walling, J. R., \& McEvoy, C. L. Doubts about depth. Journal of Experimental Psychology: Human Learning and Memory, 1979, 5, 24-44.
Perfetti, C. A., Lindsey, R., \& Garson, B. Association and uncertainty: Norms of association to ambiguous words. Pittsburgh: University of Pittsburgh, Learning Research and Development Center, 1971.

Roget's International Thesaurus (3rd ed.). New York: Crowell, 1962.

Schvaneveldt, R. W., \& Meyer, D. E. Retrieval and comparison processes in semantic memory. In $S$. Kornblum (Ed.), Attention and performance VI. New York: Academic Press, 1973.

Wollen, K. A., Cox, S. D., Coahran, M. M., Shea, D. S., \& KIRBY, R. F. Frequency of occurrence and concreteness ratings of homograph meanings. Behavior Research Methods \& Instrumentation, 1980, 12, 8-15.

(Received for publication January $24,1980$. ) 